\title{
A diferença na qualidade de vida de pacientes com doença venosa crônica leve e grave
}

\author{
Differences in the quality of life of patients with mild and severe chronic \\ venous disease
}

\author{
Raymundo Fagner Farias Novais dos Santos ${ }^{1}$, Gustavo José Martiniano Porfírio², \\ Guilherme Benjamin Brandão Pitta ${ }^{3}$
}

\section{Resumo}

Contexto: A doença venosa crônica atinge os indivíduos em sua fase mais produtiva da vida, acarretando dor, perda de mobilidade e afastamento de atividades, podendo interferir diretamente na qualidade de vida. Seus sintomas clínicos decorrem de um estado de hipertensão venosa, causada por refluxo e/ou obstrução venosa.

Objetivo: Determinar a diferença na qualidade de vida de pacientes com doença venosa crônica leve e grave.

Métodos: Foi realizado estudo transversal analítico comparativo na unidade de saúde durante 8 meses. A amostra foi calculada em 88 pacientes e dividida em dois grupos: Grupo A (CEAP clínico 1, 2 e 3) e Grupo B (CEAP clínico 4, 5 e 6). Foi usado o questionário genérico Medical Outcomes Study - 36-Item Short-Form Health Survey (SF-36). A análise estatística para verificar se havia diferença foi através do teste $t$ de Student, com intervalo de confiança de $95 \%$ e $\mathrm{p}<0,05$.

Resultados: Foram analisados 88 indivíduos, sendo 47 no grupo A e 41 no grupo B. Do total, 87,5\% (77) eram mulheres. Com relação à idade, 34\% (30) tinham entre 30 e 40 anos. As alterações subcutâneas (CEAP clínico 4) foram os sintomas mais presentes, em 28,4\%. Com exceção do domínio estado geral da saúde, todos os outros mostraram que o grupo B apresentava escores inferiores quando comparado ao grupo $\mathrm{A}$.

Conclusões: A qualidade de vida dos pacientes com doença venosa crônica leve e grave é diferente, apresentando-se mais afetada de acordo com a gravidade.

Palavras-chave: Qualidade de vida, varizes, insuficiência venosa.

\section{Introdução}

A doença venosa crônica de membros inferiores é caracterizada por um estado de hipertensão do sistema venoso. Essa hipertensão, que é causada por uma insuficiência e/ou obstrução do sistema venoso profundo, torna-se a ver-

\begin{abstract}
Background: Chronic venous disease afflicts individuals in their most productive years, resulting in pain and loss of mobility and independence in performing routine daily activities, which may directly interfere with the patient's quality of life. Clinical symptoms result from venous hypertension caused by reflux and/or venous blockage.
\end{abstract}

Objective: To determine differences in the quality of life of patients with mild and severe chronic venous disease.

Methods: A cross-sectional comparative study was conducted in a health care facility during an 8 -month period. The sample was composed of 88 patients divided into two groups: group A (clinical CEAP class 1, 2 and 3) and group B (clinical CEAP class 4, 5 and 6). The Medical Outcomes Study - 36-Item Short-Form Health Survey (SF-36) questionnaire was used. Statistical analysis was conducted using the Student $t$ test, with a $95 \%$ confidence interval and a level of significance set at $\mathrm{p}<0.05$.

Results: We analyzed 88 subjects, 47 in group A and 41 in group B. Of the total, $87.5 \%$ (77) were women and $34 \%$ (30) were aged 30 to 40 years. Subcutaneous changes (CEAP class 4$)$ were the most common symptoms, observed in $28.4 \%$ of the individuals. Except for the general health perceptions domain, group B showed lower scores than group A in all other domains.

Conclusions: The quality of life of patients with mild and severe chronic venous disease proved to be different according to disease severity.

Keywords: Quality of life, varicose veins, venous insufficiency.

dadeira responsável pelo surgimento dos sintomas clínicos da doença.

A partir da terceira década de vida, observa-se aumento significativo na incidência de doença venosa crônica,

1. Acadêmico de Medicina, Faculdades Integradas Aparício Carvalho (FIMCA), Porto Velho, RO.

2. Fisioterapeuta, Universidade Estadual de Ciências da Saúde de Alagoas (UNCISAL), Maceió, AL.

3. Doutor, Professor adjunto, Departamento de Cirurgia, UNCISAL, Maceió, AL.

Este trabalho foi realizado na Universidade Estadual de Ciências da Saúde de Alagoas (UNCISAL), Maceió, AL.

O projeto do presente estudo foi apresentado, na condição de pôster, no 37ํㅡ Congresso Brasileiro de Angiologia e Cirurgia Vascular (CBACV), em 2007.

Financiamento: Programa de Bolsas de Iniciação Científica (PROBIC), concedido pela Fundação de Amparo a Pesquisa de Alagoas (FAPEAL), AL.

Não foram declarados conflitos de interesse associados à publicação deste artigo.

Artigo submetido em 03.11.08, aceito em 18.02.09.

J Vasc Bras. 2009;8(2):143-147.

Copyright (C) 2009 by Sociedade Brasileira de Angiologia e de Cirurgia Vascular 
que atinge o indivíduo em plena maturidade, afetando diretamente sua capacidade de trabalho ${ }^{1}$. Na Europa, 5 a $15 \%$ de adultos entre 30 e 70 anos de idade, apresentam essa doença, sendo que $1 \%$ apresenta úlcera ${ }^{2,3}$. Em torno de 7 milhões de pessoas têm doença venosa crônica nos EUA, o que é responsável por cerca de 70 a $90 \%$ das úlceras de estase em membros inferiores ${ }^{2,3}$.

No Brasil, em estudo epidemiológico realizado em Botucatu (SP) foi constatada uma prevalência de 35,5\% de varizes e formas graves de doença venosa crônica, sendo que $1,5 \%$ apresentavam úlcera aberta ou cicatrizada ${ }^{4}$. Essa alta incidência é acompanhada por um custo substancial para seu tratamento. A doença venosa crônica geralmente significa dor e perda da mobilidade e independência funcional, gerando impacto direto na qualidade de vida, apresentando-se inferior nesses pacientes ${ }^{5-7}$.

O CEAP consiste no diagnóstico mais completo e classifica a gravidade clínica e a incapacidade para o trabalho no paciente com doença venosa crônica ${ }^{8}$. Além do critério clínico e anatômico, analisa também os critérios etiológicos e patológicos. A classificação clínica é dividida em: $\mathrm{C}_{0}$ (sem sinais de doença venosa), $\mathrm{C}_{1}$ (telangiectasias e veias reticulares), $C_{2}$ (veias varicosas), $C_{3}$ (edema), $C_{4}$ (alterações subcutâneas; divide-se em $\mathrm{C}_{4 a}$, que representa alterações na pigmentação e eczema, e $\mathrm{C}_{4 b}$, lipodermatoesclerose e atrofia branca) $\mathrm{C}_{5}$ (úlcera de estase cicatrizada) e $\mathrm{C}_{6}$ (úlcera de estase aberta).

A valorização do conceito de qualidade de vida reflete num aumento da preocupação com o paciente, que agora é visto como um todo. O objetivo do tratamento deixa de ser a cura da patologia e passa a ser a reintegração do pacientes com o máximo de condições de ter uma vida normal, ou seja, viver com qualidade e saúde. Sendo uma doença extremamente relevante, a doença venosa crônica acomete diferentes faixas etárias, afetando diretamente os níveis socioeconômicos, pois pode retirar o indivíduo de suas atividades normais, como o trabalho, e pode chegar a ponto de provocar a aposentadoria precoce desse indivíduo que se encontra em fase produtiva.

O questionário genérico Medical Outcomes Study 36-Item Short-Form Health Survey (SF-36) já foi citado na literatura internacional ${ }^{9,10}$. É composto por 36 itens que avaliam as seguintes dimensões: capacidade funcional, aspectos físicos, dor, estado geral de saúde, vitalidade, aspectos sociais, aspectos emocionais e saúde mental ${ }^{11}$.
A literatura relata dois tipos de questionários: o genérico e o específico. O genérico avalia de forma mais ampla os aspectos mais importantes e relevantes para se definir a qualidade de vida. O específico abrange apenas os aspectos mais afetados por determinadas patologias, aspectos descritos anteriormente em diversos estudos feitos com um questionário genérico.

A melhor forma de observar qual a verdadeira ação da doença venosa crônica no indivíduo e em sua capacidade socioeconômica e em seus aspectos psicológicos é avaliando sua qualidade de vida.

O estudo objetivou determinar a diferença na qualidade de vida de pacientes com doença venosa crônica leve e grave.

\section{Métodos}

O presente estudo obteve aprovação do Comitê de Ética em Pesquisa da UNCISAL sob parecer ño. 667/2007, com a permissão da direção da Unidade Básica CAIC Virgem dos Pobres e tendo como base a resolução 196/96 do Conselho Nacional de Saúde.

Foi realizado estudo transversal analítico comparativo, buscando mensurar a qualidade de vida em pacientes com diferentes estágios da doença venosa crônica durante o período de outubro de 2007 a julho de 2008.

O tamanho da amostra ${ }^{12}$ foi calculado em 88 indivíduos, considerando o desvio padrão relacionado com os domínios do SF-36 de 19,39 ${ }^{11}$, a diferença a ser detectada de 10 pontos no escore, o nível de significância de $5 \%$ e o poder de teste de $80 \%$. Foram incluídos no estudo pacientes com doença venosa crônica de qualquer etiologia classificada dentre as classes $\mathrm{C}_{1}$ a $\mathrm{C}_{6}$ da classificação $\mathrm{CEAP}$, que estavam na faixa etária entre 30 e 70 anos de idade, onde a patologia tem maior ocorrência e sem restrição de sexo. Não puderam participar do estudo pacientes gestantes, com déficit cognitivo e de raça indígena. Antes da inclusão, foi fornecido um Termo de Consentimento Livre e Esclarecido e o mesmo foi assinado pelo indivíduo.

Os pacientes foram divididos em dois grupos alocados de forma não-probabilística e por conveniência. O grupo A era constituído dos pacientes classificados com CEAP clínico 1,2 e 3 (forma mais leve da doença); o grupo B, CEAP clínico 4, 5 e 6 (forma mais grave da doença). Todos foram avaliados por um angiologista e diagnosticados de acordo com seu CEAP. Após o diagnóstico, o questio- 
nário foi aplicado pelo pesquisador. Além do SF-36, o formulário de coleta de dados apresentava questões utilizadas para caracterizar a amostra referentes ao sexo, faixa etária, renda, nível de escolaridade e o CEAP clínico apresentado. Os escores do SF-36 foram utilizados para determinar se havia diferença significante entre os grupos. A análise estatística foi realizada através do software Statistical Package for the Social Sciences (SPSS) versão 15.0, utilizando o teste $t$ de Student e intervalo de confiança de 95\% (IC95\%), considerando valor de $\mathrm{p}<0,05$.

\section{Resultados}

Foram coletados os dados de 88 indivíduos. Dentre eles, apenas $11(12,5 \%)$ eram do sexo masculino e 77 $(87,5 \%)$ do sexo feminino. Quanto à faixa etária, 30 (34\%) tinham entre 30 e 40 anos, $25(28,4 \%)$ entre 41 e 50 anos, $21(23,9 \%)$ entre 51 e 60 anos e o restante, 11(12,5\%), entre 61 e 70 anos de idade. Com relação aos níveis de escolaridade, 49 indivíduos (55,7\%) tinham primeiro grau incompleto, outros $19(21,6 \%)$ possuíam primeiro grau completo, $12(13,6 \%)$ segundo grau incompleto e o restante, $6(6,8 \%)$, apresentava segundo grau completo. Observando-se a renda individual mensal, a maioria, $45(51,1 \%)$, ganhava no máximo R \$400,00, 14 (15,9\%) tinham renda inferior a R \$200,00, 26 (29,5\%) tinham salários de até $\mathrm{R} \$ 800,00$ e apenas os $2,3 \%$ (2) restantes superavam ganhos de $\mathrm{R} \$ 800,00$, revelando a realidade dos pacientes da unidade de saúde.

Para a variável principal, os escores do SF-36 de cada grupo foram comparados com o teste $t$ de Student, e se o valor de $\mathrm{p}$ for $<0,05$, a diferença estatística entre os grupos é considerada significante. Analisando os grupos, o grupo $\mathrm{B}$ apresentou pior qualidade de vida quando comparado ao grupo A em quase todos os domínios, com exceção do estado geral de saúde (ver Tabela 1). Os domínios capacidade funcional, limitação por aspectos físicos, vitalidade, aspectos sociais e dor apresentaram um valor de $p<0,0001$, ou seja, extremamente significante. O domínio limitação por aspectos emocionais $(p=0,0230)$ e saúde mental $(\mathrm{p}=0,0014)$ também se mostraram inferiores a 0,05 , sendo considerados estatisticamente relevantes. Já o estado geral de saúde, com valor de $\mathrm{p}=0,0593$, apesar de próximo do limite, não pode ser considerado relevante (Tabela 1). O grupo B obteve escores inferiores ao grupo A, com exceção do item estado geral de saúde.

Com relação à frequência da classificação CEAP, foi utilizado o intervalo de confiança, que quanto menor o intervalo, mais significativa a amostra. Observando o CEAP clínico, com 28,4\% (25 indivíduos, com IC95\% 20,0-38,6\%) as alterações subcutâneas $\left(\mathrm{C}_{4}\right)$ mostraram-se mais frequentes dentre os que procuraram a unidade básica de saúde (ver Tabela 2). Seguida de veias varicosas $\left(\mathrm{C}_{2}\right)$, com 22,7\% (20 indivíduos, com IC95\% 15,1-32,6\%), telangiectasias $\left(\mathrm{C}_{1}\right)$ com $17 \%$ (15 indivíduos, com IC95\% 10,5-26,3\%), úlcera de estase cicatrizada $\left(\mathrm{C}_{5}\right)$ com 15,9 (14), edema $\left(\mathrm{C}_{3}\right)$ apresentando $13,6 \%(12)$ e úlcera de estase aberta $\left(\mathrm{C}_{6}\right)$ com apenas $2,3 \%$ (2) (demais valores expressos na Tabela 2).

\section{Discussão}

Um estudo realizado no Brasi $1^{1}$, mostrou que a incidência da doença venosa crônica no sexo feminino é supe-

Tabela 1 - Comparação entre os grupos através dos domínios do SF-36

\begin{tabular}{lccc}
\hline Domínios do SF-36 & Grupo A (DP) & Grupo B (DP) & p \\
\hline Capacidade funcional & $75,1(11,5)$ & $47,3(13,5)$ & $<0,0001$ \\
Limitação por aspectos físicos & $70,7(24,6)$ & $28,0(17,8)$ & $<0,0001$ \\
Dor & $32,3(8,6)$ & $15,1(6,4)$ & $<0,0001$ \\
Estado geral da saúde & $46,1(13,1)$ & $39,1(19,0)$ & 0,0593 \\
Vitalidade & $52,7(12,5)$ & $40,2(13,3)$ & $<0,0001$ \\
Aspectos emocionais & $73,8(25,0)$ & $61,0(26,8)$ & 0,023 \\
Aspectos sociais & $78,6(12,7)$ & $58,1(16,3)$ & $<0,0001$ \\
Saúde mental & $60,9(8,7)$ & $53,2(12,1)$ & 0,0014 \\
\hline
\end{tabular}

$\mathrm{DP}=$ desvio padrão; SF-36 = Medical Outcomes Study - 36-Item Short-Form Health Survey.

Os valores entre parênteses correspondem ao desvio padrão das médias expostas para cada domínio. Os dados são considerados estatisticamente significantes quando $\mathrm{p}<0,05$. 
Tabela 2 - Frequência da classificação clinica do CEAP

\begin{tabular}{lccc}
\hline Classificação clínica & $\mathrm{n}$ & Frequência (\%) & IC95\% \\
\hline $\mathrm{C}_{1}$ & 15 & 17,1 & $10,5-26,3$ \\
$\mathrm{C}_{2}$ & 20 & 22,7 & $15,1-32,6$ \\
$\mathrm{C}_{3}$ & 12 & 13,6 & $7,8-22,4$ \\
$\mathrm{C}_{4}$ & 25 & 28,4 & $20,0-38,6$ \\
$\mathrm{C}_{5}$ & 14 & 15,9 & $9,6-25,0$ \\
$\mathrm{C}_{6}$ & 2 & 2,3 & $0,1-8,4$ \\
Total & 88 & 100,0 & - \\
\hline
\end{tabular}

IC95\% = intervalo de confiança de 95\%.

rior, apresentando-se em $45 \%$ de uma população normal. Neste estudo, $88 \%$ dos participantes eram do sexo feminino, também com incidência superior quando comparado com o masculino.

Mesmo em países desenvolvidos, a incidência da doença venosa crônica é significativa ( 5 a $15 \%$ em adultos $)^{2}$. Quando comparada com a de países em desenvolvimento, como no caso do Brasil ${ }^{4}$, vê-se que cerca de 35,5\% apresentam algum estágio da doença.

Constatando os baixos índices de qualidade de vida, um estudo prospectivo randomizado ${ }^{13}$ notou que mesmo após uma intervenção cirúrgica, os níveis de qualidade de vida não melhoraram significativamente durante um período de 2 anos, confirmando o impacto da doença também notado nesta pesquisa. Além de ressaltar escores de qualidade de vida semelhantes aos obtidos aqui, outro estudo realizado na Turquia ${ }^{14}$ mostrou indícios de que a localização anatômica da insuficiência e/ou obstrução venosa também pode ter relação.

Os índices de dor, função física e mobilidade já foram citados como os mais afetados pela doença venosa crôni$\mathrm{ca}^{15}$, semelhante aos resultados encontrados.

Um estudo multicêntrico ${ }^{16}$ em países desenvolvidos revelou que a forma mais incidente de doença venosa crônica encontrada é a presença de veias varicosas associadas a alterações de pele, veias varicosas com presença de edema e telangiectasias, respectivamente.

Refletindo sobre as atuais políticas de saúde, que buscam cuidar da integralidade do indivíduo, a qualidade de vida é um aspecto importante e vem a explicitar índices que mais se assemelham com seu estado de saúde real. Em uma revisão sistemática ${ }^{17}$, o estágio mais avançado da do- ença venosa crônica teve seu impacto comprovado na qualidade de vida, sendo significante na diminuição de seus índices, devido à cronicidade e ao fato de prejudicar os indivíduos em idade produtiva.

No Brasil $^{18}$, a deterioração da qualidade de vida em pacientes com úlcera venosa é clara, sugerindo a importância de uma equipe multidisciplinar no tratamento, já que se tem um impacto tanto no aspecto físico quanto no psicológico. Ainda há a carência de estudos que possam definir uma situação da doença venosa crônica nacionalmente, mas baseando-se em estudos internacionais de grande impacto ${ }^{16}$ e alguns estudos nacionais, nota-se que a qualidade de vida é diretamente afetada pela doença e a crescente necessidade por promoção da qualidade de vida nesses indivíduos.

\section{Conclusão}

A qualidade de vida nos pacientes com doença venosa crônica leve e grave é diferente. Os indivíduos que apresentam a forma mais grave da doença tiveram menores índices nos escores do SF-36. Itens como dor, aspectos físicos, aspectos sociais e emocionais, vitalidade, saúde mental e capacidade funcional mostraram-se significativamente mais afetados em indivíduos com estágio mais avançado da doença.

\section{Referências}

1. Maffei FHA. Insuficiência venosa crônica: diagnóstico e tratamento clínico. In: Maffei FHA, Lastória S, Yoshida WB, Rollo HA, Giannini M, Moura R. Doenças vasculares periféricas. Rio de Janeiro: Guanabara Koogan; 2008.

2. Brand FN, Dannenberg AL, Abbott RD, Kannel WB. The epidemiology of varicose veins: the Framingham study. Am J Prev Med. 1988;4:96-101.

3. Heit JA, Rooke TW, Silverstein MD, et al. Trends in the incidence of venous stasis syndrome and venous ulcer: a 25-year population-based study. J Vasc Surg. 2001;33:1022-7.

4. Maffei FHA, Magaldi C, Pinho SZ. Varicose veins and chronic venous insufficiency in Brazil: prevalence among 1755 inhabitants of a country town. Int $\mathrm{J}$ Epidemiol. 1986;15:210-7.

5. Miller WL. Chronic venous insufficiency. Curr Opin Cardiol. 1995;10:543-8.

6. Ibrahim S, Macpherson DR, Goldhaber SZ. Chronic venous insufficiency: mechanisms and management. Am Heart J. 1996;132:856-60.

7. Kan YM, Delis KT. Hemodynamic effects of supervised calf muscle exercise in patients with venous leg ulceration. Arch Surg. 2001;136:1364-9.

8. Eklöf B, Rutherford RB, Bergan JJ, et al. Revision of the CEAP classification for chronic venous disorders: consensus statement. J Vasc Surg. 2004;40:1248-52. 
9. McHorney CA, Ware JE, Raczek AE. The MOS 36-item short-form health survey (SF-36): II. Psychometric and clinical tests of validity in measuring physical and mental health constructs. Med Care. 1993;31:247-63.

10. Ware JE Jr, Sherbourne D. The MOS 36-item short-form health survey (SF-36). I. Conceptual framework and item selection. Med Care. 1992;30:473-83.

11. Ciconelli RM, Ferraz MB, Santos W, Meinão I, Quaresma MR. Tradução para a língua portuguesa e validação do questionário genérico de avaliação de qualidade de vida SF-36 (Brasil SF-36). Rev Bras Reumatol. 1999;39:143-50.

12. Lwanga SK, Lemeshow S. Sample size determination in health studies: a practical manual. Geneva: World Health Organization; 1991.

13. Blomgren L, Johansson G, Bergqvist D. Quality of life after surgery for varicose veins and the impact of preoperative duplex: results based on a randomized trial. Ann Vasc Surg. 2006;20:30-4.

14. Sadikoglu G, Ozcakir A, Ercan I, Yildiz C, Sadikoglu Y. Does the anatomical localization of lower extremity venous disease affect the quality of life? Saudi Med J. 2006;27:1683-7.

15. Bergan JJ, Schmid-schönbein GW, Smith PDC, Nicolaides AN, Boisseau MR, Eklöf B. Chronic venous disease. Minerva Cardioangiol. 2007;55:459-76.

16. Kurz X, Lamping DL, Kahn SR, et al. Do varicose veins affect quality of life? Results of an international populationbased study. J Vasc Surg. 2001;34:641-8.

17. Herber OR, Schnepp W, Rieger MA. A systematic review on the impact of leg ulceration on patients' quality of life. Health Qual Life Outcomes. 2007;5:44.

18. Longo Jr O, Buzatto SHG, Fontes AO, Miyazaki COM, Godoy JMP. Qualidade de vida em pacientes com lesões ulceradas crônicas na insuficiência venosa de membros inferiores. Cir Vasc Angiol. 2001;17:21-6.

Correspondência:

Raymundo Fagner Farias Novais dos Santos

Rua Dep. Rubens Canuto, 215/101 - Ponta Verde

CEP 57035200 - Maceió, AL

Tel.: (82) 8803.4087

E-mail: rayfagner@hotmail.com 